\title{
Recommendations to improve smoking cessation outcomes from people with lung conditions who smoke
}

\author{
Sarah Masefield ${ }^{1}$, Pippa Powell ${ }^{1}$, Carlos Jiménez-Ruiz ${ }^{2}$, Peter Hajek ${ }^{3}$, \\ Keir Lewis ${ }^{4}$, Stefan Andreas ${ }^{5}$, Philip Tønnesen ${ }^{6}$, Onno van Schayck ${ }^{7}$, \\ Christina Gratziou ${ }^{8}$, Bertrand Dautzenberg ${ }^{9}$, Serena Tonstad ${ }^{10}$, \\ Thomas Hering $^{11}$, Stephano Nardini ${ }^{12}$ and Monica Fletcher ${ }^{13}$
}

Affiliations: ${ }^{1}$ European Lung Foundation, Sheffield, UK. ${ }^{2}$ Institute of Public Health, Smoking Cessation Clinic, Madrid, Spain. ${ }^{3}$ Wolfson Institute of Preventive Medicine, Queen Mary University of London, London, UK. ${ }^{4}$ Prince Philip Hospital and Swansea College of Medicine, Dept of Respiratory Medicine, Wales, UK. ${ }^{5}$ Dept of Cardiology and Pneumology, Lungenfachklinik Immenhausen, Immenhausen, Germany. ${ }^{6}$ Gentofte Hospital, Pulmonary Medicine, Hellerup, Denmark. ${ }^{7}$ Care and Public Health Research Institute (Caphri), Maastricht University, General Practice, Maastricht, The Netherlands. ${ }^{8}$ Evgenidio Hospital, Smoking Cessation Centre, Athens, Greece. ${ }^{9}$ Groupe Hospitalier Pitie-Salpetriere, Pulmonology, Paris, France. ${ }^{10}$ Department of Preventive Cardiology, Ulleval University Hospital, Oslo, Norway. ${ }^{11}$ Stellvertretender Vorsitzender des Bundesverbandes der Pneumologen, Lungenarztpraxis Tegel, Berlin, Germany. ${ }^{12}$ Ospedale Civile, Pulmonary and TB Unit, Vittorio Veneto, Italy. ${ }^{13}$ Education for Health, Warwick, UK.

Correspondence: Sarah Masefield, European Lung Foundation, 442 Glossop Road, Sheffield, S10 2PX. E-mail. Sarah.masefielddeuropeanlung.org

ABSTRACT This study aimed to gain insight into the impact of lung conditions on smoking behaviour and smoking cessation, and identify recommendations for smoking cessation and professional-patient communications. The study was led by the European Lung Foundation in collaboration with the European Respiratory Society Task Force on "Statement on smoking cessation on COPD and other pulmonary diseases and in smokers with comorbidities who find it difficult to quit".

A web-based observational cross-sectional questionnaire was developed from a patient-centered literature review. Topics covered were: cohort characteristics; perspectives on smoking cessation; interactions with healthcare professionals; and recommendations to improve cessation outcomes.

The questionnaire was disseminated via existing patient and professional networks and social media channels. The survey was available online for a period of 4 months in 16 languages. The data were analysed as a whole, not by country, with thematic analysis of the open responses.

Common characteristics were: male (54\%); age 40-55 years (39\%); 11-20 cigarettes a day (39\%); smokes within $30 \mathrm{~min}$ of waking (61\%); and has made 1-5 cessation attempts in the previous 12 months (54\%). $59 \%$ had tried cessation treatments, but, of these, 55\% had not found any treatments helpful.

Recommendations were: earlier intervention; discussion of the patient's smoking beliefs, behaviours and motivation; giving constructive advice; understanding addiction; informed decision-making; and treatment options. Areas for new and further research have been highlighted through exploring the smoking cessation perspectives and recommendations of people with lung conditions in Europe who smoke.

@ERSpublications

People across Europe with lung conditions and who smoke make recommendations to improve smoking cessation outcomes http://ow.ly/10wJGC

This article has supplementary material available from openres.ersjournals.com

Received: Jan 262016 | Accepted after revision: March 282016

Conflict of interest: M. Fletcher was European Lung Foundation chair at the time of the study. S. Masefield and P. Powell are employees of the European Lung Foundation. Further disclosures can be found alongside this article at openres. ersjournals.com

Copyright $\odot$ ERS 2016. This article is open access and distributed under the terms of the Creative Commons Attribution Non-Commercial Licence 4.0. 


\section{Introduction}

Smoking leads to $>650000$ premature deaths in Europe every year, with estimates that $\sim 1$ billion people worldwide will be killed by tobacco in the 21st century [1]. Smoking is the leading cause of chronic obstructive pulmonary disease (COPD), with projections that, by 2020, COPD will have become the third cause of death and the fifth cause of disability worldwide [2]. Although only $15 \%$ of the world's population live in Europe, it carries nearly a third of the burden of tobacco-related disease, with a huge impact on national economies and healthcare services. To combat this growing health crisis, effective smoking-cessation services, delivered and signposted by healthcare professionals (HCPs) are essential $[1,3]$.

The number of premature deaths and the impact of lung disease on quality of life and symptom control can be reduced by raising awareness of the value of smoking cessation to people with a lung condition, such as COPD, asthma and lung cancer [2-5]. For example, smoking cessation is the only effective means of controlling the progression of COPD [2, 3, 6, 7]; however, $40 \%$ of people with COPD continue to smoke $[3,8]$. HCPs are in a position to engage with this population, but little research has been conducted into the smoking cessation behaviours and perspectives of people with lung conditions in Europe, including their perspectives on smoking-related interactions with HCPs and cessation services.

There is a growing understanding that a single "one size fits all" approach to smoking cessation with this complex population does not work, with calls for further characterisation of this smoking population and for a more individualised approach to their needs, and to smoking cessation in general [7, 9-13].

The objectives of this study were to explore characteristics of people across Europe with a diagnosed lung condition and who currently smoke; their perceptions of cessation services and treatments; their interactions with HCPs; and their recommendations for improving cessation outcomes. This study was led by the European Lung Foundation (ELF) in collaboration with the European Respiratory Society (ERS) Task Force on "Statement on smoking cessation on COPD and other pulmonary diseases and in smokers with comorbidities who find it difficult to quit" [3].

\section{Methods}

\section{Literature review}

PubMED, CINAHL and keyword internet searches were used. Studies published in English, or with English translations, exploring populations in European countries, published in 2000-2015 were included. The search parameters were defined as: 1) lung disease; 2) current smoking; 3) smoking cessation; and 4) patient perspectives. These included the search terms: 1) smoking-related, respiratory/lung diseases/conditions, chronic obstructive pulmonary disease, COPD, asthma, lung cancer; 2) chronic/persistent/long-term/ involuntary/active/hardcore/recalcitrant smoking/smoker; 3) smoking cessation, stop smoking clinic, difficulty quitting/stopping, unable to quit, hard to stop; and 4) perspectives, beliefs, perceptions, experiences.

The majority of quantitative smoking cessation and lung disease research looks at COPD, focusing on cessation rates and clinical observations on specific treatments, with minimal feedback from the participants in the studies [6, 14-17]. Similarly, the most relevant qualitative research comprises of small sample size interview and questionnaire designs conducted with people with COPD [9, 18-22]. There has been little wider investigation into common factors for people with lung diseases and transcending European national contexts has been conducted [3, 14, 16, 23-25].

Due to the limited literature, studies on related topics were also considered: characteristics of people who find it difficult to stop smoking; challenges to smoking cessation in people without lung conditions [13-15, 17, 18, 20, 24-30]. Relevant factors identified were lifestyle and environment, individual smoking experience, relationship between lung disease and smoking, desire to stop smoking and impact of repeated cessation attempts.

The descriptor "people with a lung condition who smoke" is used throughout. Questionnaire respondents who consented to being contacted with additional questions and entered an email address considered the descriptor to be clear and non-judgemental.

\section{Questionnaire}

An explorative study design and sampling strategy was selected as most appropriate for gaining insight into this hard-to-reach population. A web-based observational questionnaire was developed from the literature review, with the questions refined by a sub-group of the ERS Task Force, including smoking cessation and respiratory medicine clinicians, researchers and a psychologist. The 18 -item questionnaire covered four main topics: cohort characteristics; perspectives on smoking cessation; interactions with healthcare professionals; and recommendations to improve cessation outcomes. The draft questionnaire was reviewed by two people with lung conditions who smoke to ensure the appropriate level and tone. 
The questionnaire (see supplementary material) was available in 16 languages (English, German, French, Spanish, Italian, Greek, Polish, Russian, Dutch, Turkish, Bulgarian, Serbian, Portuguese, Romanian, Swedish and Norwegian). The questionnaire was open from December 2012 to March 2013. Full details of the aim of the questionnaire, anonymity and contact details for the ELF were provided at the start of the questionnaire, with implied consent given by each person choosing to complete the questionnaire [31].

The online format enabled rapid and wide-reaching dissemination of the questionnaire across Europe, using existing ELF electronic communication channels, such as the ELF website with an Adwords campaign in each language, the ELF newsletter and social media. However, this approach prevented participation by patients without internet access at home or in their doctor's clinic. Information and promotional posters were also sent to ERS members and to ELF's European patient organisation network to share with their colleagues, patients and members.

This snowball approach to sampling, in order to reach the maximum number of potential questionnaire respondents, facilitated the engagement of diverse patients but made it impossible to record the number of people aware of the questionnaire and who chose not to respond [31, 32]. Furthermore, due to inconsistency in the recording of smoking status across Europe, the potential size of the target population is also unknown [23]. For these reasons neither the response rate nor the generalisability of the findings relative to the size of the population could be calculated. However, the findings are validated by the similarities between the sample and the European respiratory patient population, thus giving reliability and generalisability to the findings and to the areas highlighted for further study.

The information entered into the anonymous questionnaire was considered truthful, as there was no incentive for inaccurate reporting, and studies have found no statistically significant difference in patient self-reported smoking behaviour and tobacco consumption as measured with cortisone testing [33, 34]. The data were analysed as a whole, not by country, and expressed using descriptive statistics. No maximum/minimum number of responses for language or country was set; therefore, comparisons between countries could not be made. Open responses were translated into English. Thematic analysis explored commonalities. The STROBE (Strengthening the Reporting of Observational Studies in Epidemiology) checklist for observational studies was applied [35].

\section{Results}

In total, 514 questionnaires were completed, with 490 responses from 26 European countries included in the study. Questionnaires were excluded from 21 respondents resident outside Europe, two respondents without diagnosed lung conditions, one person who had successfully stopped smoking, and one who did not complete the respondent characteristic questions (numbers 15-17). The highest responses by country were Turkey (22\%), UK (21\%) and Poland (20\%).

\section{Characteristics of people with lung conditions}

Common respondent characteristics were: male (54\%), aged 40-55 years (39\%), with COPD (70\%). Asthma was the second most common condition (23\%). A further 20 conditions were stated, including tuberculosis (6\%), bronchiectasis (5\%), lung cancer (4\%), sarcoidosis (2\%) and pulmonary fibrosis (2\%), and 18 others and comorbidities (17\%). Respondents could indicate more than one condition.

Levels of consumption and dependence were gauged by how much the respondents smoked per day and how soon after waking [36]. 76\% of respondents smoked $1-20$ cigarettes per day $(1-10,37 \% ; 11-20,39 \%)$ within $30 \mathrm{~min}$ of waking (61\%) ( $\leqslant 5 \mathrm{~min}, 23 \%$; 6-30 $\mathrm{min}, 38 \%$ ).

Impact of diagnosis on smoking behaviour was highlighted by changes in the amount smoked or enjoyment of smoking. 59\% of respondents reported smoking less since diagnosis, $34 \%$ smoked the same amount and 7\% smoked more since diagnosis. Most respondents enjoyed smoking less (52\%) or to the same extent (44\%), with $4 \%$ enjoying smoking more since diagnosis.

\section{Experiences of smoking cessation}

Common reasons for continuing to smoke were: "it helps me cope with stress" (49\%); "I tried to quit but do not seem able to do it" (43\%); and "I enjoy smoking" (40\%) (figure 1). 10\% of respondents gave additional reasons. Habit was the most common additional reason, with catastrophic thinking evident in some cases: "I think that if I stop smoking my world would simply collapse". Some focused on the impact of stress and environment, such as managing difficult family situations, isolation and boredom, or held beliefs that inhibited cessation, such as weight loss or gain, helping loosen mucus or reduce constipation. Others stated that it was too late or that cessation would cause an earlier death.

In the previous 12 months, $54 \%$ had made 1-5 attempts to stop smoking, $33 \%$ had made no attempts, and $4 \%$ had made $>20$ attempts. Different approaches to cessation were preferred, with $55 \%$ thinking that 


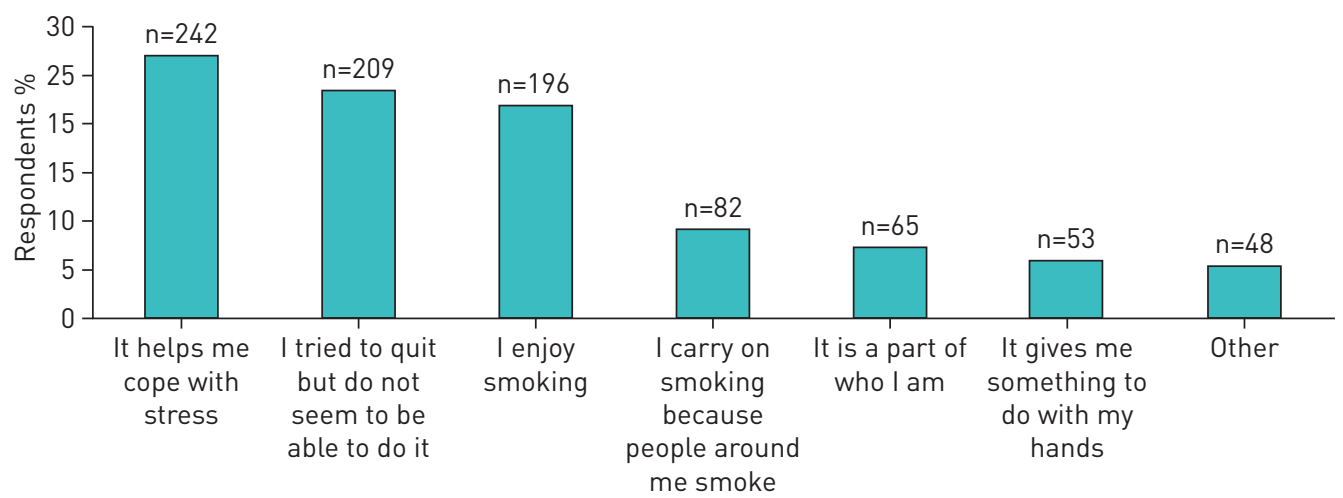

FIGURE 1 Main reasons for survey respondents not stopping smoking (multiple choices could be selected).

setting a date to stop smoking and stopping abruptly had been more successful for them, whilst $45 \%$ thought trying to reduce smoking with cessation as a longer-term goal worked better for them. Although the majority showed a desire to stop smoking, 90\% found it "very difficult" or "difficult" to stop.

$60 \%$ of the respondents had tried cessation treatments, including prescription and over-the-counter drugs, alternative therapies and self-help resources. However, only $40 \%$ of the entire cohort of respondents reported that these treatments helped them to stop smoking (figures 2 and 3). The most common treatment tried was nicotine replacement therapy (NRT), such as patches, chewing gum and inhalers (31\%) (figure 2). Electronic cigarettes (e-cigarettes; 20\%) and self-help books (19\%) were also popular. Additional treatments tried were identified by $12 \%$ of respondents, including will power, mouth spray (non-NRT), chewing sweets and mints, cytisine (Tabex) and reducing smoking. 37\% of respondents had tried two or more treatments, ranging from 2-10 treatments tried.

The respondents that had tried treatments reported finding NRT (12\%) and e-cigarettes (11\%) the most helpful in helping them stop smoking (figure 3). However, only $6 \%$ found self-help books helpful (figure 3 ). Other treatments found helpful by $11 \%$ of respondents were cytisine (Tabex), smoking reduction, the shock and physical impact of their diagnosis, and will power.

\section{Interactions with HCPs}

Respondents were asked when doctors or nurses had talked to them about smoking, and their expectations and feelings about the interaction. The majority reported that doctors/nurses had talked to them about smoking both before and after their diagnosis (41\%). $31 \%$ had a conversation following diagnosis. $15 \%$ had never had a conversation with an HCP about smoking or only before their diagnosis (13\%).

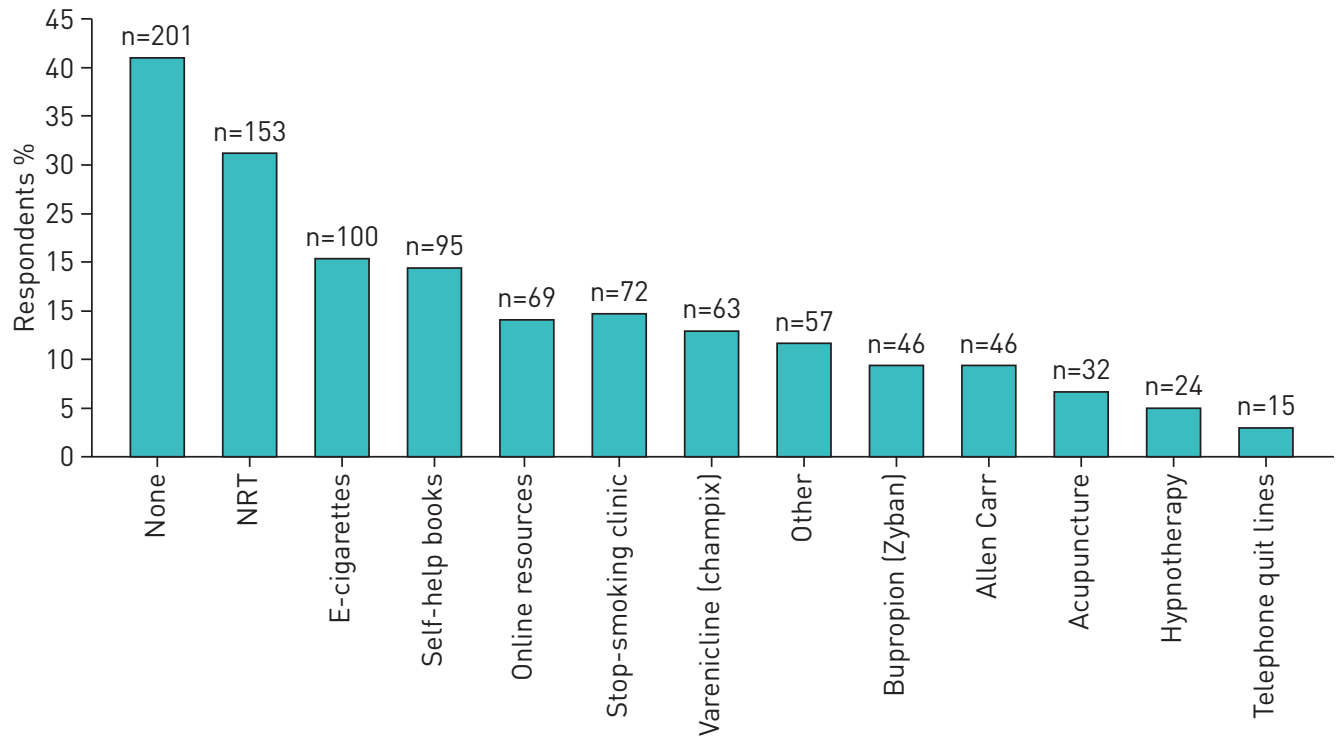

FIGURE 2 Smoking treatments tried (percentage of the cohort that had tried each treatment). Multiple choices could be selected. NRT: nicotine replacement therapy. 


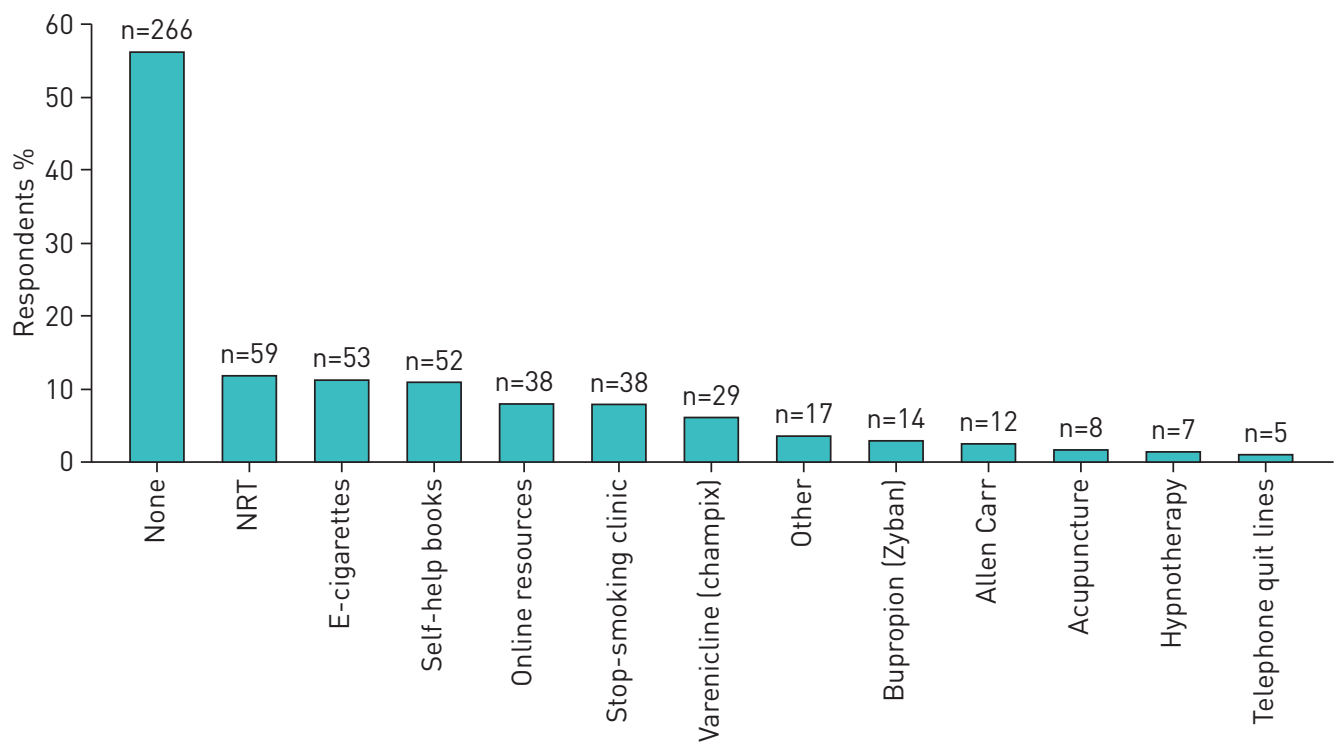

FIGURE 3 Smoking treatments found most helpful (percentage of the cohort that found each treatment helpful). Multiple choices could be selected. NRT: nicotine replacement therapy.

The most common statements were "I was hoping they could help me stop smoking" (27\%), "I felt they were nagging/lecturing" (24\%), "I felt they are being helpful" (23\%), "I did not think they could do much about it" (22\%), and "I was happy to discuss [smoking]" (22\%). Only 9\% felt that smoking was "raised too often".

\section{Recommendations to improve cessation outcomes}

Recommendations based on six key themes are illustrated with indicative quotations.

\section{Systemic improvement}

Respondents recommended longer consultations or scheduled follow-up appointments for discussion and monitoring of cessation attempts. Longer appointments would give HCPs time to explain the effects of smoking, benefits of cessation for their lung condition and treatment options. For example: "an explanation of what smoking does to your body and your lungs, plus a good proposal about how to stop smoking would be welcome"; "give time to discuss consequences and how to cope effectively". The respondents wanted to feel like collaborators in their treatment, which is only possible if HCPs explain the impact of smoking on an individual's lung disease and outline therapy options to enable informed decision-making. Respondents stated: "I need more information"; "giving out brochures with an explanation from the doctor, not just putting a bundle of paper in your hands".

A range of treatment options should be available, especially when standard treatments have not helped achieve cessation, including alternative therapies. For example: "I have tried patches, tablets, the whole range, nothing has worked, maybe some new approach, hypnotherapy or anything really that could help"; "rather than discussing pharmacological agents to stop smoking, they should discuss more about the methods which do not require pharmacological agents".

Respondents requested access to cessation services and a specialist, including in-patient clinics and stop-smoking courses and reduction or cessation plans. HCPs telling respondents to stop smoking without offering further guidance or support reduced their motivation to try to stop smoking and resulted in unsuccessful attempts. For example: "I would be elated to receive some help, rather than just being told 'you must give up"; "there are lots of tips but not much practical help".

\section{Understanding addiction}

Respondents referred to the addictive nature of tobacco and the need to treat it like alcohol and non-prescription drug addictions. Many felt that HCPs do not adequately understand nicotine addiction and the difficulty stopping: "I feel they could be more appreciative of how hard it is to quit... No one wants to smoke but the fact is unfortunately we are addicted!"

Many found it hard to take advice from a doctor or nurse that they know smokes, whilst others found it difficult to take advice from someone who does not smoke and cannot understand the addiction: "if you have never been a smoker, it must be hard to understand how you could continue with such an obviously harmful habit". 


\section{Empathy}

Respondents wanted signs of empathy, for HCPs to "show some understanding of the difficulties of giving up smoking". Many felt "embarrassed", "looked down on as an addict" or treated as less intelligent: "we are smokers NOT idiots. Yes, smoking kills us BUT WE KNOW THAT! We just find it very difficult to stop. Don't patronise us, consider how we feel". One respondent summarised the message stated by many: HCPs should "never speak dogmatically or patronisingly. Empathy and compassion in the particular situation means there is a chance that the patient will feel more comfortable and therefore will be ready to talk seriously about the issue".

\section{Individualised treatment}

Respondents called for HCPs to consider their personal relationship with smoking, rather than prescribing a standard cessation treatment: "each patient must be treated individually, not everyone is the same; as with diseases, so with smoking, the success of the treatment involves a subjective element". Underlying stressors and reasons for continuing to smoke should be discussed, "find out the reasons why people don't stop smoking rather than putting all the emphasis on why we smoke".

Many identified smoking as a complex psychological issue and need for holistic treatment: "to look at the whole picture of what is going on in someone's life-to help deal with the reasons someone smokes (i.e. as stress relief) and then build up to a cessation attempt". The relationship between smoking, stress and challenging environments/circumstances was evident in the responses, with smoking maintained by "the stress of work and my daughter", "the severely challenging factors in my working and personal environments".

\section{Personal responsibility}

Many respondents identified the choice to smoke and to stop as the individual's responsibility: "Support or drugs or counselling is needed, but it's up to me and only me"; "the final decision rests with me and I don't appear to have the will power to overcome this addiction". HCPs could discuss smoking with people with lung conditions, providing information and a number of treatment options, alongside helping develop the individual's self-efficacy so that they can support themselves in cessation attempts.

\section{Greater public awareness}

Greater public awareness of the health risks of tobacco was considered a necessary early intervention to dissuade people from starting and continuing to smoke, to "better emphasise the damage smoking does before you get a lung disease!". Some felt that the general population is not well-informed about tobacco-related lung conditions and would not consider the longer-term risks when starting to smoke. Awareness-raising political and social interventions were suggested, such as social policy to affect change, billboards advertising the dangers of smoking, public lectures and awareness events to educate about smoking as a health problem.

\section{Discussion}

The responses to the online questionnaire are consistent with the literature on the characteristics of people with lung conditions who smoke, and current guidelines on smoking cessation for people who find it difficult to stop smoking [3, 8, 28, 29]. Respondents were not asked about their socio-economic or education status. However, the open responses regarding smoking beliefs, motivation and number of quit attempts are consistent with qualitative research into smoking in low socio-economic groups [18, 19, 34], thus validating the study methodology used and giving generalisability to the findings and areas highlighted for further study.

A key finding is that $67 \%$ of respondents made at least one cessation attempt in the last 12 months, and either smoke less (59\%) or enjoy smoking less (52\%) since their diagnosis. This indicates a desire to stop smoking and supports the recommendation that HCPs should frequently repeat cessation advice, as there may be a period following diagnosis when the person is more receptive to cessation guidance $[1,3]$. ERS guidance for HCPs states that "patients with respiratory disease have a greater and more urgent need to stop smoking than the average smoker, so respiratory physicians must take a proactive and continuing role with all smokers in motivating them to stop" [3].

In exploring the smoking cessation experiences and recommendations of people with lung conditions who smoke, areas for new and further research have been highlighted with reference to supporting literature, where available (table 1). These include understanding of addiction, psychological and holistic approaches, combinations and variety of treatments, inpatient facilities, e-cigarettes and HCP's communication styles.

The aim of the study was achieved by providing an explorative insight into the cohort of people with lung conditions who smoke across Europe, their perspectives of smoking cessation and interactions with HCPs. The study has shown a motivation by people with lung conditions who smoke to use their experiences to help improve cessation services. The questionnaire methodology was successful in reaching a hard-to-reach 
TABLE 1 Recommendations to improve smoking cessation outcomes with supporting literature, where available

It is important for the healthcare professional to understand $[6,18,25]$ each person's level of addiction and the high level of challenge in attempting to stop smoking

Frequently repeat cessation advice and plan follow-up visits to address smoking cessation as the primary issue

Discussing reasons for relapse can provide important information for tailoring individual treatments

Self-efficacy and self-esteem affect the individual's ability to stop smoking, so advice should be targeted to address issues of motivation, self-efficacy and self-esteem

Pharmacological and psychological support should be provided as part of smoking cessation treatment due to the frequency of anxiety, depression and panic disorders

Healthcare professionals should be empathic, understanding and show respect to the individual's opinions about smoking

If a certain treatment does not help, offer a new or different cessation treatment

People who find it especially difficult to stop smoking due to their home environment could benefit from intensive treatment, such as in an inpatient cessation facility for an agreed period of time

If someone finds it difficult to stop smoking abruptly, i.e. $[10,13,14,18]$ "going cold turkey", suggest a reduction programme over an agreed period of time

Earlier public and individual intervention, such as public awareness initiatives, and routine discussion of smoking is needed, especially before a lung disease has developed

Allow time to discuss the individual's relationship with smoking and reasons for continuing

Give constructive advice on cessation strategies and support

Give a range of treatment options, and explain them so that each person can make an informed decision about their treatment

Explain the effect of smoking and how stopping would affect each person's lung condition

population across Europe, and has highlighted specific areas for investigation, including in national populations or disease-specific patient sub-groups and using a dissemination approach that will track the number of people invited to participate and produce a response rate. By building on the findings of this study the recommendations and experiences of people with lung conditions who still smoke can be used to inform individual services, national smoking cessation strategies and European guidelines to achieve more successful cessation attempts.

\section{Acknowledgements}

We would like to thank the European Respiratory Society and the Task Force on "Consensus on smoking cessation on COPD and other pulmonary diseases" for supporting the European Lung Foundation (ELF) in conducting this study to explore patient perspectives in smoking cessation. ELF would also like to thank everyone who completed the online questionnaire and provided additional recommendations and feedback on their experiences.

\section{References}

1 Tønnesen P. Tobacco smoking. In: Gibson GJ, Loddenkemper R, Sibille Y, et al., eds. European Lung White Book. 2nd Edn. Sheffield, European Respiratory Society, 2013.

2 Underner M, Perriot J, Peiffer G. Smoking cessation in smokers with chronic obstructive pulmonary disease. Rev Mal Respir 2014; 31: 937-960.

3 Jiménez-Ruiz CA, Andreas S, Lewis KE, et al. Statement on smoking cessation in COPD and other pulmonary diseases and in smokers with comorbidities who find it difficult to quit. Eur Respir J 2015; 46: 61-79.

4 Raupach T, Quintel M, Hinterthaner M. Preoperative smoking cessation in patients with lung cancer. Pneumologie 2010; 64: 694-700. 
5 Thomson NC, Chaudhuri R. Asthma in smokers: challenges and opportunities. Curr Opin Pulm Med 2009; 15: 39-45.

6 Qaseem A, Wilt TJ, Weinberger SE, et al. Diagnosis and Management of Stable Chronic Obstructive Pulmonary Disease: A Clinical Practice Guideline Update from the American College of Physicians, American College of Chest Physicians, American Thoracic Society, and European Respiratory Society. Ann Intern Med 2011; 155: 179-191.

7 Jones D. Chronic obstructive pulmonary disease part 1: smoking cessation. Nurs Stand 2015; 29: 52-57.

8 Global Strategy for the Diagnosis, Management and Prevention of COPD, Global Initiative for Chronic Obstructive Lung Disease (GOLD). Global strategy for the diagnosis, management, and prevention of COPD 2016. www.goldcopd.org/uploads/users/files/GOLD_Report\%202016.pdf Date last updated: December, 2015. Date last accessed: January 15, 2016.

9 Eklund BM, Nilsson S, Hedman L, et al. Why do smokers diagnosed with COPD not quit smoking? A qualitative study. Tob Induc Dis 2012; 10: 17.

10 Yap SY, Lunn S, Pang E, et al. A psychological intervention for smoking cessation delivered as treatment for smokers with chronic obstructive pulmonary disease: Multiple needs of a complex group and recommendations for novel service development. Chron Respir Dis 2015; 12: 230-237.

11 van Eerd EA, van Rossem CR, Spigt MG, et al. Do we need tailored smoking cessation interventions for smokers with COPD? A comparative study of smokers with and without COPD regarding factors associated with tobacco smoking. Respiration 2015; 90: 211-219.

12 Gratziou C, Florou A, Ischaki E, et al. Smoking cessation effectiveness in smokers with COPD and asthma under real life conditions. Respir Med 2014; 108: 577-583.

13 Ritchie D, Schulz S, Bryce A. One size fits all? A process evaluation - the turn of the 'story' in smoking cessation. Public Health 2007; 121: 341-348.

14 Coronini-Cronberg S, Heffernan C, Robinson M. Effective smoking cessation interventions for COPD patients: a review of the evidence. JRSM Short Reports 2011; 2: 78 .

15 Strong DR, Leventhal AM, Evatt DP, et al. Positive reactions to tobacco predict relapse after cessation. J Abnorm Psychol 2011; 120: 999-1005.

16 Balmford J, Borland R, Hammond D, et al. Adherence to and reasons for premature discontinuation from stop-smoking medications: data from the ITC Four-Country Survey. Nicotine Tob Res 2011; 13: 94-102.

17 Jones D. Chronic obstructive pulmonary disease part 2: non-pharmacological therapy. Nurs Stand 2015; 29: 53-58

18 Medbø A, Melbye H, Rudebeck CE. "I did not intend to stop. I just could not stand cigarettes anymore." A qualitative interview study of smoking cessation among the elderly. BMC Fam Pract 2011; 12: 42.

19 Walters JA, Cameron-Tucker H, Courtney-Pratt H, et al. Supporting health behaviour change in chronic obstructive pulmonary disease with telephone health-mentoring: insights from a qualitative study. BMC Fam Pract 2012; 13: 55 .

20 Bethea J, Murtagh B, Wallace SE. "I don't mind damaging my own body" a qualitative study of the factors that motivate smokers to quit. BMC Public Health 2015; 15: 4.

21 Schofield I, Kerr S, Tolson D. An exploration of the smoking-related health beliefs of older people with chronic obstructive pulmonary disease. J Clin Nurs 2007; 16: 1726-1735.

22 Wilson JS, Elborn JS, Fitzsimons D. 'It's not worth stopping now': why do smokers with chronic obstructive pulmonary disease continue to smoke? A qualitative study. J Clin Nurs 2011; 20: 819-827.

23 Bogdanovica I, Godfrey F, McNeill A, et al. Research paper. Smoking prevalence in the European Union: a comparison of national and transnational prevalence survey methods and results. Tob Control 2011; 20: e4.

24 Borland R, Yong HH, Balmford J, et al. Motivational factors predict quit attempts but not maintenance of smoking cessation: findings from the International Tobacco Control Four country project. Nicotine Tob Res 2010; 12: 4-11.

25 Vangeli E, Stapleton J, Smit ES, et al. Predictors of attempts to stop smoking and their success in adult general population samples: a systematic review. Addiction 2011; 106: 2110-2121.

26 Kerr S, Watson $\mathrm{H}$, Tolson D, et al. Smoking after the age of 65 years: a qualitative exploration of older current and former smokers' views on smoking, stopping smoking, and smoking cessation resources and services. Health Social Care Community 2006; 14: 572-582.

27 Argüder E, Hasanoğlu HC, Karalezli A, et al. Factors that increasing tendency to smoking cessation. TuberkToraks 2012; 60: 129-135.

28 Zernig G, Wallner R, Grohs U, et al. 'A randomized trial of short psychotherapy versus sustained-release bupropion for smoking cessation.' Addiction 2008; 103: 2024-2031.

29 Marino MG, Fusconi E, Magnatta R, et al. Epidemiologic determinants affecting cigarette smoking cessation: a retrospective study in a National Health System (SSN) treatment service in Rome (Italy). J Environ Public Health 2010; 2010: 183206.

30 Wang D, Connock M, Barton P, et al. 'Cut down to quit' with nicotine replacement therapies in smoking cessation: a systematic review of effectiveness and economic analysis. Health Technol Assess 2008; 12: 1-135.

31 Bowling A. Research Methods in Health: Investigating Health and Health Services. 4th Edn. Maidenhead, Open University Press, 2014.

32 Shaghaghi A, Bhopal RS, Sheikh A. Approaches to recruiting 'hard-to-reach' populations into research: a review of the literature. Health Promot Perspect 2011; 1: 86-94.

33 Ramo DE, Hall SM, Prochaska JJ. Reliability and validity of self-reported smoking in an anonymous online survey with young adults. Health Psychol 2011; 30: 693-701.

34 Studts JL, Ghate SR, Gill JL, et al. Validity of self-reported smoking status among participants in a lung cancer screening trial. Cancer Epidemiol Biomarkers Prev 2006; 15: 1825-1828.

35 Von Elm E, Altman DG, Egger M, et al. STROBE Initiative. The Strengthening the Reporting of Observational Studies in Epidemiology (STROBE) statement: guidelines for reporting observational studies. J Clin Epidemiol 2008; 61: 344-349.

36 Fagerström K, Furberg H. A comparison of the Fagerström Test for Nicotine Dependence and smoking prevalence across countries. Addiction 2008; 103: 841-846. 